\title{
Abnormal Activity Detection for the Elderly People using ConvLSTM Autoencoder
}

\section{Ehsan Nazerfard ( $\nabla$ nazerfard@gmail.com )}

Amirkabir University of Technology Department of Computer Engineering and Information Technology https://orcid.org/0000-0003-2649-3440

\section{Zahra Atashgahy}

Amirkabir University of Technology

\section{Alireza Nadali}

Amirkabir University of Technology

\section{Research Article}

Keywords: Machine Learning, Smart Homes, Anomaly Detection, ConvLSTM, Autoencoder

Posted Date: July 20th, 2021

DOl: https://doi.org/10.21203/rs.3.rs-693084/v1

License: (c) (1) This work is licensed under a Creative Commons Attribution 4.0 International License. Read Full License 


\title{
Abnormal activity detection for the elderly people using ConvLSTM Autoencoder
}

\author{
Ehsan Nazerfard, Zahra Atashgahy, Alireza Nadali \\ \{nazerfard, zahraatashgahy, a_nadali\}@aut.ac.ir \\ Department of Computer Engineering, Amirkabir University of Technology, No. 350, Hafez Ave, \\ Valiasr Square, Tehran, Iran
}

\begin{abstract}
Independent living for elderly people is often viewed as an impossible task, due to the great many perils and difficulties. With advancements of Ambient Intelligence, this scenario is no longer out of reach and smart homes offer a computationally inexpensive solution to this problem. In this paper we address these difficulties and propose a novel method for Anomaly Detection in elderly's daily routine and behavior. Our proposed model is a ConvLSTM Autoencoder for processing spatiotemporal data, given the fact that these type of behavior and anomalies are sparse and rare, it is safe to presume anomalies are harder to recreate in Autoencoder and have a much higher reproduction error compared to normal behavior. We utilized two datasets from the WSU CASAS smart home project to validate our proposed method by comparing it to the other state-of-the-art approaches.
\end{abstract}

Keywords: Machine Learning, Smart Homes, Anomaly Detection, ConvLSTM, Autoencoder

\section{Introduction}

Studies show that by the end of 2030 , more than $3 \%$ of the population will be aged between 65 and 74. One of the common cognitive impairments in elderly people is Dementia, when it damages the brain to the point that one is unable to perform everyday activities. Currently, there is no cure and no treatment that slows or stops its progression. Here in this study, we would like to detect the early symptoms of dementia in order to alert the patient's caregiver. Dementia in early stages can make complicated chores and daily tasks more cumbersome. Furthermore, Alzheimer's disease accounts for over $80 \%$ of cases of dementia worldwide [1]. People with Alzheimer's usually suffer from short-term memory loss, which may cause anomalies in their behavior.

Anomaly or outlier detection is the pinpointing of observations that are different significantly from the majority of dataset. Anomaly detection methods are usually employed when the majority of data in the training dataset is normal, but the available "Anomaly" data are insufficient to perform a supervised learning and utilize its methods. 
Moreover, in real-world applications, "Labels" are not available at all and it is not feasible nor possible to acquire labels for the dataset. In general, signals generated by people suffering from Dementia have characteristic patterns that are significantly different from normal. In our case, the behavior itself may not be considered as anomaly, but repetition or time frame makes it an outlier, like having a meal around midnight.

In recent years, Deep Learning has revolutionized field of Artificial Intelligence. Researchers around the world managed to achieve results that were previously inconceivable [2]. The abundance of data in our era made methods and algorithms based on Deep Learning truly shine. Anomaly detection methods based on Deep Learning methods or Deep Anomaly detection techniques learn hierarchical discriminative features from the data. Anomalies are essentially classified into three types: point anomalies, contextual anomalies and collective anomalies. Deep anomaly detection methods have had a great success distinguishing these anomalies.

One of the most successful Anomaly Detection methods is One-Class Support Vector Machine (OCSVM) which is an unsupervised method that combines the uncertainty information and the importance information in order to detect anomalous data. Later in the paper we compare our results with this algorithm and generally, there are major improvement in the context of Ambient Intelligence.

The rest of the work is organized as follows: In section 2, we briefly review the related work and Anomaly Detection in different fields. Then in section 3, we provide preliminaries required to follow our proposed method. Next, we describe our proposed method and architecture in section 4 . We then present the experimental results in section 5. Finally in section 6 , we close the paper by providing some concluding remarks.

\section{Related work}

Anomaly Detection is particularly useful in industry [3] since labeled data is unavailable and the exact model of a system is not accurate due to aging and small differences in manufacturing. In financial domain, Anomaly detection is mainly used for fraud detection in different areas such as Healthcare, Financial Services and manufacturing [4]. Deng et al. [5] leveraged self-organizing maps and k-means clustering in order to detect fraudulent financial statements.

Anomaly detection is still at early stages in smart homes and much more developed in other areas as we mentioned above, yet it has been the center of many studies. Shin et al. [6] showed promising results using Support Vector Data Description in detecting anomalies in living patterns for elderly people who live alone. Also there are studies which leveraged different machine learning methods such as Naïve Bayes [7], Restricted Boltzmann Machines (RBMs) [8] and Markov logic networks [9]. In recent years, there has been a growing interest in Deep Learning techniques and methods, mostly due to the abundance of data and how well these methods perform when there is sufficient data to train, however, there is a lack of deep learning based anomaly detection in smart homes. 
Recently, researchers all around the world have shown great interest in Convolutional Neural Networks (CNNs) [10,11, 12] and Recurrent Neural Networks (RNNs) [13] in human activity recognition. Some of these studies take advantage of the inherent properties of CNNs and Long Short Term Memories (LSTMs) in activity recognition [12]. Moreover, deep convolutional and recurrent architectures have been used in order to analyze and process movement data with wearable sensors [13]. Although CNNs have achieved great success in activity recognition, there are not many studies that focus on activity detection in smart homes. Arifoglu et al. [14] utilized these architectures in a supervised manner in activity and abnormal behavior detection. In this work, we employ Autoencoder together with ConvLSTM cells in order to perform anomaly detection the Aruba and Kyoto daily activity datasets, both from WSU CASAS smart home project [15].

\section{Preliminaries}

In this section, we provide some preliminaries to make it easier to follow the description of our proposed model.

\subsection{Recurrent Neural Networks}

Recurrent Neural Network (RNN) is a type of artificial neural networks that is used to process sequential data, which allows modeling temporal dynamic behavior. In Artificial Neural Networks, one may presume that data instances are independent but in activity detection, this presumption does not hold true. RNNs have internal states which makes it possible to process sequential data. They are typically as follows (see Fig. 1):

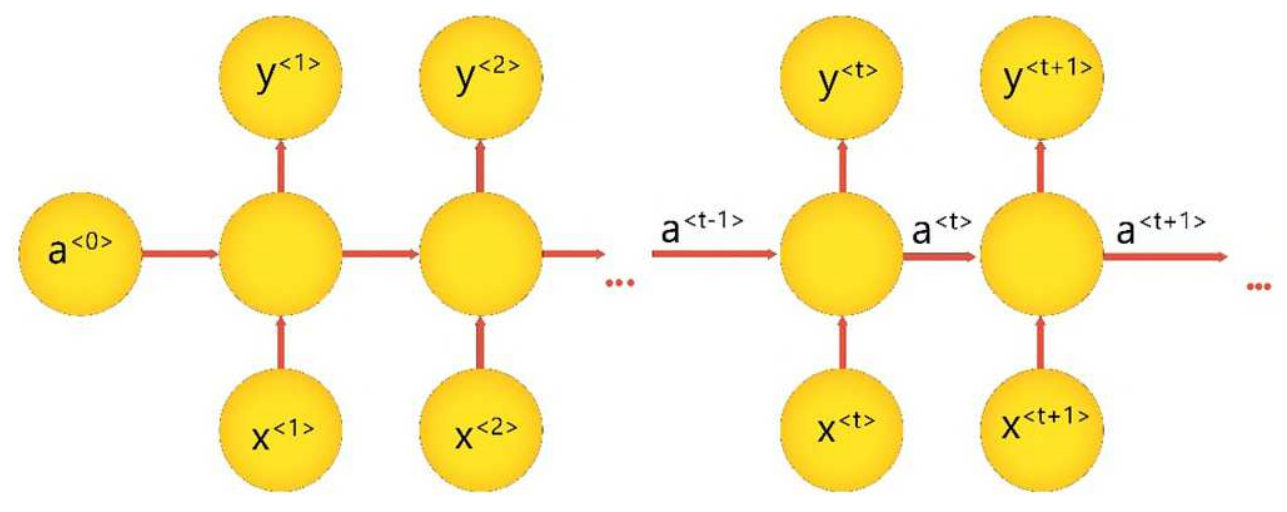

Fig. 1: Schematic representation of a traditional RNN

For each timestep $t$, the activation $a^{<t>}$ and the output $y^{<t>}$ are expressed as follows:

$$
\begin{gathered}
a^{<t>}=g_{1}\left(W_{a a} a^{<t-1>}+W_{a x} x^{<t>}+b_{a}\right) \\
y^{<t>}=g_{2}\left(W_{y a} a^{<t>}+b_{y}\right),
\end{gathered}
$$

where $W_{a a}, W_{a x}, W_{y a}, b_{a}, b_{y}$ denote coefficients that are shared temporally and $g_{1}, g_{2}$ are activation functions. 


\section{2. $L S T M$}

Long short-term memory networks [16] were introduced in 1997 and outperformed traditional RNN models [17]. It is shown that for Artificial Neural Networks (ANNs) trained with any gradient-based method, the error is likely to vanish as it travels back through space or time, due to the fact that as it travels back, it is multiplied by scaling factor, which results in vain weight updates. In order to tackle this problem, LSTMs use a memory cell containing a self-connected linear unit, thus allowing it to maintain relatively unscaled error derivatives in different time-steps. Each LSTM memory block contains input gate, forget gate and output gate (see Fig. 2). These cells are connected recurrently to one another.

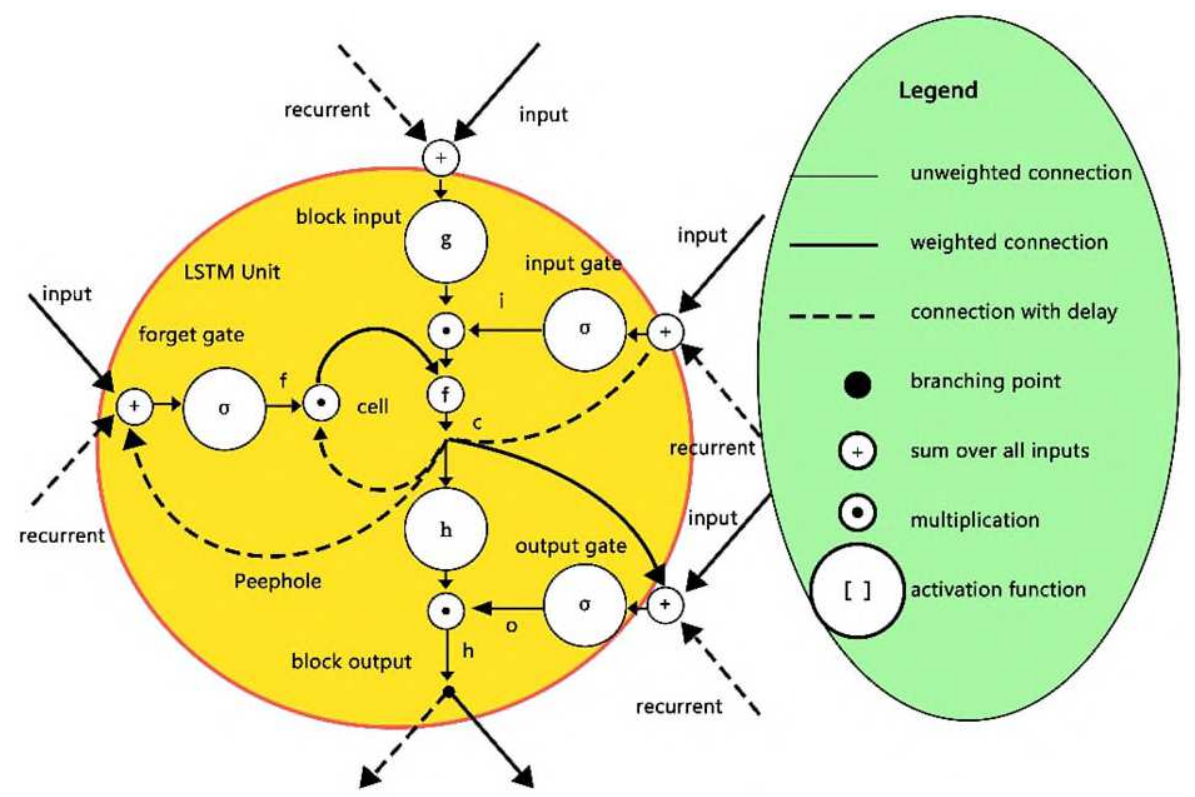

Fig. 2: Schematic representation of an LSTM unit

The equations in (1) denote the mechanism to update an LSTM unit at time $t$, where $\sigma$ is the sigmoid function and $\odot$ is the element-wise product. Also, $x_{t}$ and $h_{t}$ denote the input and hidden state vector at time $t$, respectively. Also, $W^{z}, W^{i}, W^{f}, W^{o}$ denote the weight matrices of different gates for input $x_{t}$. Moreover, $R^{z}, R^{i}, R^{f}, R^{o}$ are recurrent weights and $b^{z}, b^{i}, b^{f}, b^{o}$ denote bias vector.

\begin{tabular}{rr}
\hline$z_{t}=\tanh \left(W^{z} x_{t}+R^{z} h_{t-1}+b^{z}\right)$ & (input) \\
$i_{t}=\sigma\left(W^{i} x_{t}+R^{i} h_{t-1}+b^{i}\right)$ & (input gate) \\
$f_{t}=\sigma\left(W^{f} x_{t}+R^{f} h_{t-1}+b^{f}\right)$ & (forget gate) \\
$o_{t}=\sigma\left(W^{o} x_{t}+R^{o} h_{t-1}+b^{o}\right)$ & (output gate) \\
$s_{t}=z_{t} \odot i_{t}+s_{t-1} \odot f_{t}$ & (cell state) \\
$h_{t}=\tanh \left(s_{t}\right) \odot o_{t}$ & (output)
\end{tabular}




\subsection{Convolutional Neural Networks}

A convolutional Neural Network (CNN) [18] is a type of a deep neural networks, most commonly used to process data with spatial correlation such as images. CNNs have been tremendously successful in computer vision. As the name implies, CNNs employ convolution operator in at least one of their layers. CNNs consist of Convolutional layers and pooling layers. In convolutional layers, one may use different kernels in order to perform convolution. In pooling layers, results from previous convolutional layer is subsampled (typically using a max function) to extract most relevant features. After this, extracted features are fed to a fully connected layers in order to perform classification.

\subsection{ConvLSTM}

Nowadays it is quite common to find spatiotemporal data, like videos, satellite pictures or security cameras. The major downside of using LSTM for processing spatiotemporal data is redundancy and lack of leveraging the spatial information. ConvLSTM [19] is a recurrent layer, but the inputs, cell outputs and hidden states and gates are 3D tensors, which is depicted in Fig. 3.

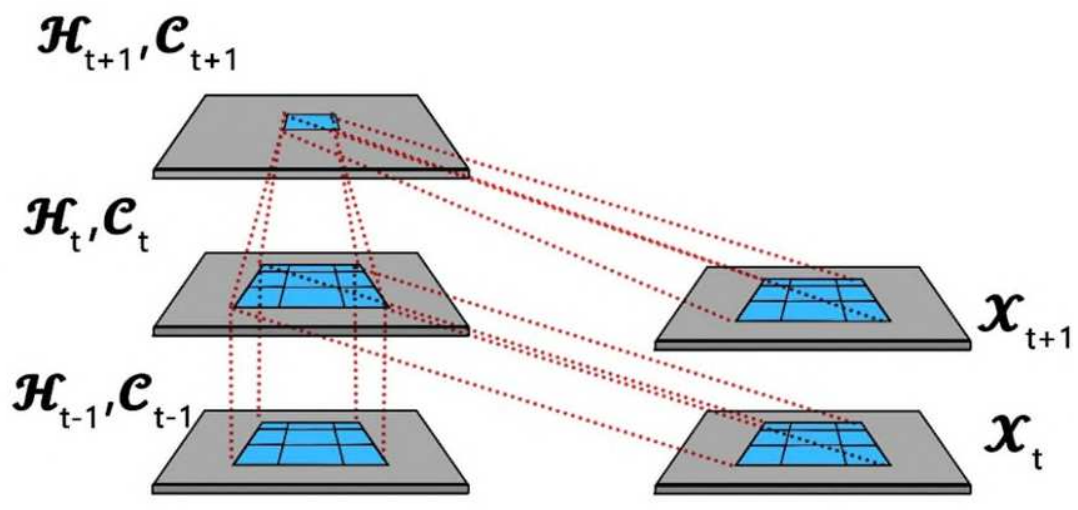

Fig. 3: Internal structure of a ConvLSTM

Equations governing the ConvLSTM cells are shown in (2), where '*' denotes the convolution operator and 'o' element-wise product.

$\boldsymbol{i}_{t}=\sigma\left(W_{x i} * \chi_{t}+W_{h i} * \mathcal{H}_{t-1}+W_{c i} \circ \mathcal{C}_{t-1}+b_{i}\right)$
$\boldsymbol{f}_{t}=\sigma\left(W_{x f} * \chi_{t}+W_{h f} * \mathcal{H}_{t-1}+W_{c f} \circ \mathcal{C}_{t-1}+b_{f}\right)$
$\mathcal{C}_{t}=\boldsymbol{f}_{t} \circ \mathcal{C}_{t-1}+i_{t} \circ \tanh \left(W_{x c} * \chi_{t}+W_{h c} * \mathcal{H}_{t-1}+b_{c}\right)$
$o_{t}=\sigma\left(W_{x o} * \chi_{t}+W_{h o} * \mathcal{H}_{t-1}+W_{c o} \circ \mathcal{C}_{t-1}+b_{o}\right)$
$\mathcal{H}_{t}=o_{t} \circ \tanh \left(\mathcal{C}_{t}\right)$

\subsection{Autoencoders}

An autoencoder (AE) is a neural network that is trained to reproduce the input as its output [20]. Autoencoders have a hidden layer $h$ that tried to represent input in another domain. Autoencoders consist of two parts: an encoder function $h=f(x)$ and a decoder that reconstructs $r=g(h)$. Encoder finds a new domain to map the input data, which 
generally have a smaller dimension than $x$.

Reproducing inputs as output may seem futile, but traditionally, AEs have been used for dimensionality reduction. That being said, AEs can be utilized for Anomaly detection. We presume that Encoder learned an accurate mapping function for inputs and decoder reproduces the input, so instances with high reconstruction error are believed to be anomalies, since they cannot be mapped and then reproduced like normal instances.

\subsection{Data sets}

In this research, we employed the Aruba dataset [15] as well as the Kyoto dataset ${ }^{1}$ [7], both from the WSU CASAS smart home testbeds, in order to validate our method. The Aruba dataset, which is collected from an off-campus smart apartment, contains sensor data that was collected in the home of a volunteer adult. The resident's children and grandchildren visited on a regular basis. In this dataset, there are motion and door/cabinet sensors. The Aruba apartment layout is illustrated in Fig. 4 [21]. As it can be seen in Fig. 4, there are 35 binary valued sensors (31 motion and 4 door/cabinet sensors) and the dataset is provided as a list of (sensor, timestamp) sensor readings. Furthermore, there are 11 daily activities over the course of more than 7 months. These activities are Cook, Relax, Eat, Work, Sleep, Wash the dishes, Bed to toilet transition, Enter home, Leave home, Housekeeping and Respirate. Since the mentioned activities in the Aruba dataset are normally performed, we modified some of these activities to reflect abnormal behavior.

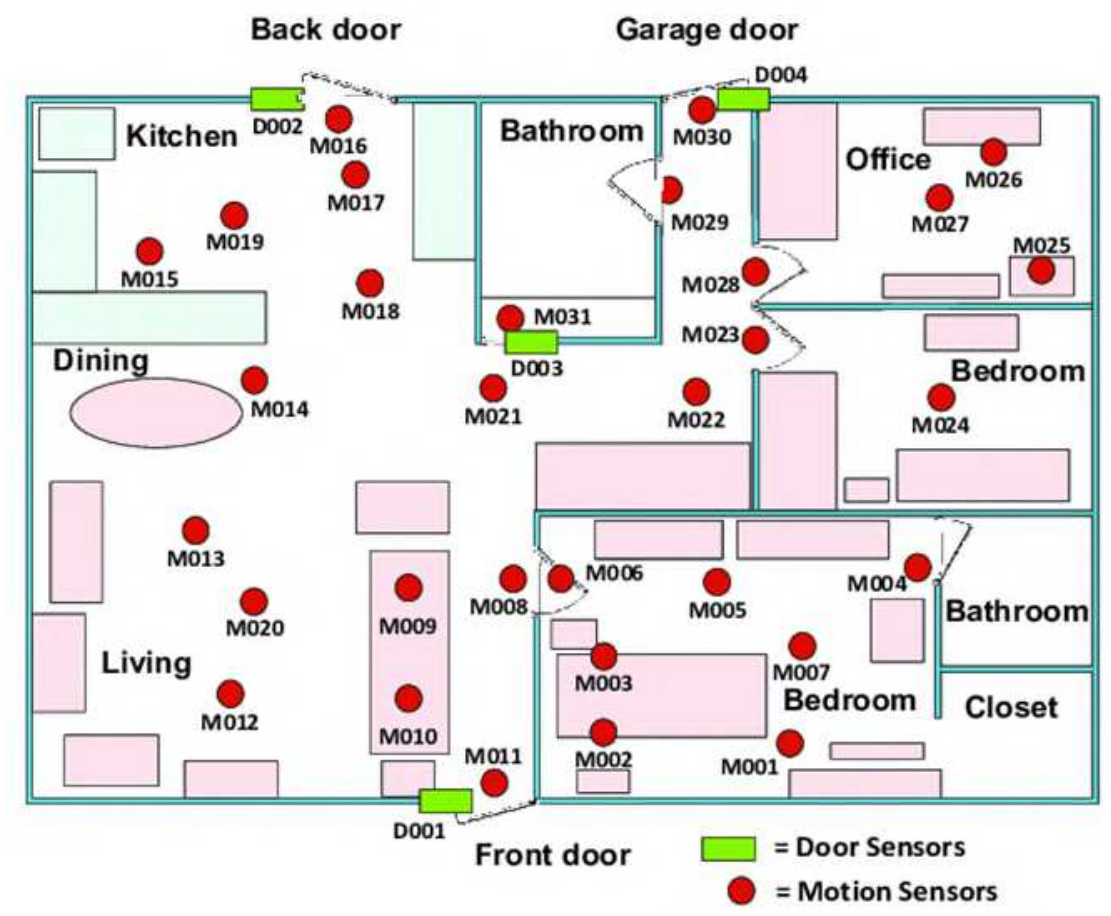

Fig. 4: The sensor layout for the Aruba apartment, where the red circles represent motion/area sensors, and the green rectangles represent door/cabinet sensors.

\footnotetext{
${ }^{1}$ Available online at http://casas.wsu.edu/datasets/
} 
In the Kyoto dataset; however, there are 5 activities performed by 20 students both in adlnormal and adlerror versions. The adlnormal version consists of normally performed activities, while in the adlerror version, there are some specific errors in completion of the activities. The errors reflect common difficulties that can compromise everyday functional independence. These errors can be seen in daily life activities of elderly people who are suffering from cognitive impairments. The activities are Make a phone call, Wash hands, Eat, Clean and Cook. The sensor layout of the Kyoto smart apartment, which is an on-campus testbed, as well as a sample for sensor events are shown in Fig. 5 [7].

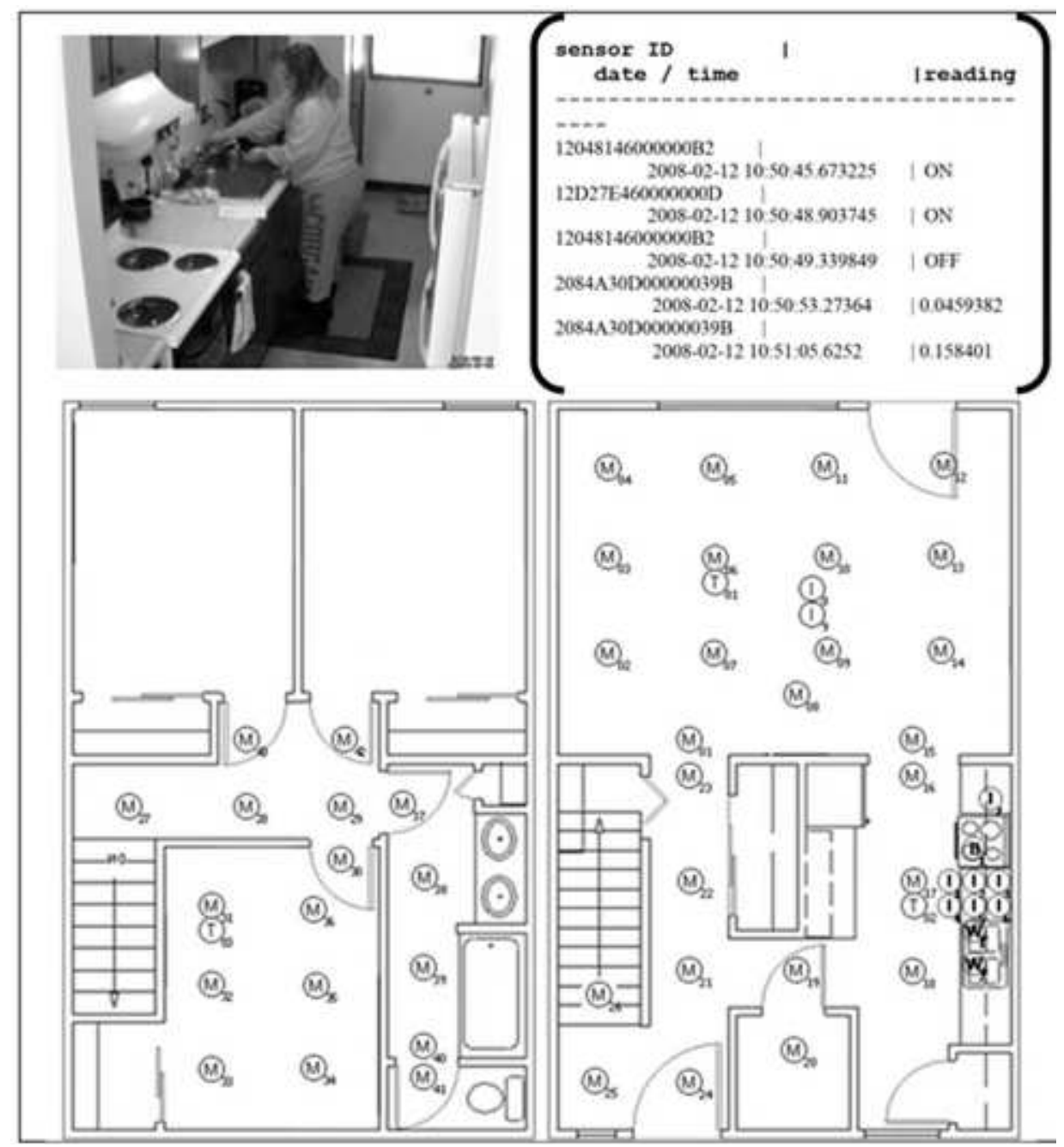

Fig. 5: A student participating in the study (top-left), a sample for sensor readings (top-right), and the sensor layout for the Kyoto smart apartment (bottom).

The description of the sensors represented in Fig. 5 can be categorized as follows:

M: motion sensors, I: item sensors for oatmeal, raisins, brown sugar, bowl, measuring spoon, medicine container, pot, and phone book, W: water sensors, and B: burner sensor. It should be added that we did not utilize the temperature sensors (denoted by $\mathrm{T}$ in Fig. 5). 
First and foremost, time-period batches are extracted without any preprocessing using sliding window approach [22]. Length of the chosen window is set to 30 seconds empirically. Then this batch is mapped into last-fired representation, meaning the sensor that its binary value changed last in the window shall output 1 until another sensor changes. It is shown that the representation presented in [23] results in better activity recognition compared to the other representations.

\subsection{Abnormal behaviors pertaining to dementia}

In this research, we seek to distinguish three abnormal behaviors from normal activities. These activities that usually are symptoms of dementia are: Repetition, Disturbance in sleep, and Confusion [14].

(1) Repetition: Elderly people who suffer from dementia are prone to forgetting whether they already performed a particular task or not. Time sensitive activities such as having a meal or drink, taking medicine, personal hygiene, etc. are such activities that only the number of incidents is of importance in medical assessments. For instance, one might forget to have lunch, have multiple lunches [22], may forget to have dinner and start preparing it in a completely irrelevant time.

In the interest of replicating this behavior, we synthesized it by manually inserting a particular set of actions in a random time of normal activities. Hence, it will result in multiple reappearance of that anomaly, happening in different periods of time. As an example, the following instance was generated presuming one forgot to have dinner: brushing teeth, sleeping, preparing dinner, eating, sleeping. Let us assume that $\mathrm{S}$ is a sequence of activities $S=d_{1} \cdot d_{2} \ldots . d_{n}$ that have occurred in a day such as here each $\mathrm{d}$ is a period of time pertaining to a certain task, then we choose an arbitrary time period (denoted as $\mathrm{x}$ ) to inset the anomalous data, so $S=d_{1} \cdot d_{2} \ldots d_{x} . a_{1} \cdot a_{2} \ldots a_{m} . d_{x+1} \ldots . d_{n}$. Anomalous data here could be any task that occurs in a specific time, like preparing dinner, brushing teeth, personal hygiene and etc. Thus we have multiple occurrences of any given activity in the sequence.

(2) Disturbance in sleep: One of the symptoms of dementia in late stages is disruption in their sleeping schedule. The older adults suffering from dementia may have significant changes in their sleeping habits and in some cases, they cannot sleep at all. They may wake up numerous times to go to the lavatory [23]. To simulate this behavior, we insert particular activities during normal night-time sequence. These activities are Eating, Bed to toilet transition and Sleeping in an arbitrary area. Again let us assume $S=n_{1} . n_{2} \ldots . n_{n}$ sequence is a sequence of night-time activities, time-slice instances of preparing dinner are injected into this sequence so $=n_{1} \cdot n_{2} \ldots n_{x} \cdot p_{1} \cdot p_{2} \ldots p_{m} . d_{x+1} \ldots . d_{n}$. Hence, we have a cooking and eating dinner activity in middle of the night. With respect the repetition and disturbance in sleep abnormal behaviors, we totally added 150 activity slices to the Aruba dataset.

(3) Confusion: Older adults who are suffering from dementia are prone to getting confused during activities. For instance, they may leave the burner on after cooking. In order to test our proposed method against these kind of abnormal behavior, we took advantage of the Kyoto dataset. The adlerror activities in this dataset consist of confusions such as forgetting the medication with the meal or leaving the water running after washing hands, so we assumed that 
the Confusion abnormal behavior is already reflected in the Kyoto dataset.

\section{Proposed Method}

We refer to our proposed model as ConvLSTMAE, short for ConvLSTM Autoencoder, for anomaly detection in daily activities. In our model, Encoder and Decoder are ConvLSTM layers as opposed to normal Convolutional Layer (see Fig. 6).

As it was previously mentioned, dataset that we leveraged for this research is spatiotemporal. Convolutional Layers in ConvLSTM process spatially correlated data, and LSTM layers process temporally correlated data. Malhorta et al. [24] developed an LSTMbased Encoder-Decoder for anomaly detection, which was the inspiration to this research, but the main assumption in their research is that data instances are not topologically correlated. Clearly, this assumption does not hold true in our datasets, hence employing ConvLSTM layers in Autoencoder. Our encoder consists of two ConvLSTM layer: First layer with 100 and second layer with 50 filters, and kernel size of 3x3, also window size is 30 seconds. Decoder is an LSTM layer since it achieved similar results compared to more complex architectures and ConvLSTM cells. Deeper and more complicated models did not affect performance, so we decided not to employ more computationally expensive models since only two layers of LSTM cells is more than enough to achieve satisfactory results.

Let us assume we have a sequence $X=\left\{X^{(1)} \cdot X^{(2)} \ldots . X^{(L)}\right\}$, where each instance is an $M \times N$ matrix which contains reading of each sensor with their corresponding location in the home. The datasets we used consists of temporal sequences only, mapping these sequences to the accompanying location, hence forming the matrix, makes it spatiotemporal. Network is first trained on normal data, minimizing the reconstruction error using Stochastic Gradient Descent algorithm.

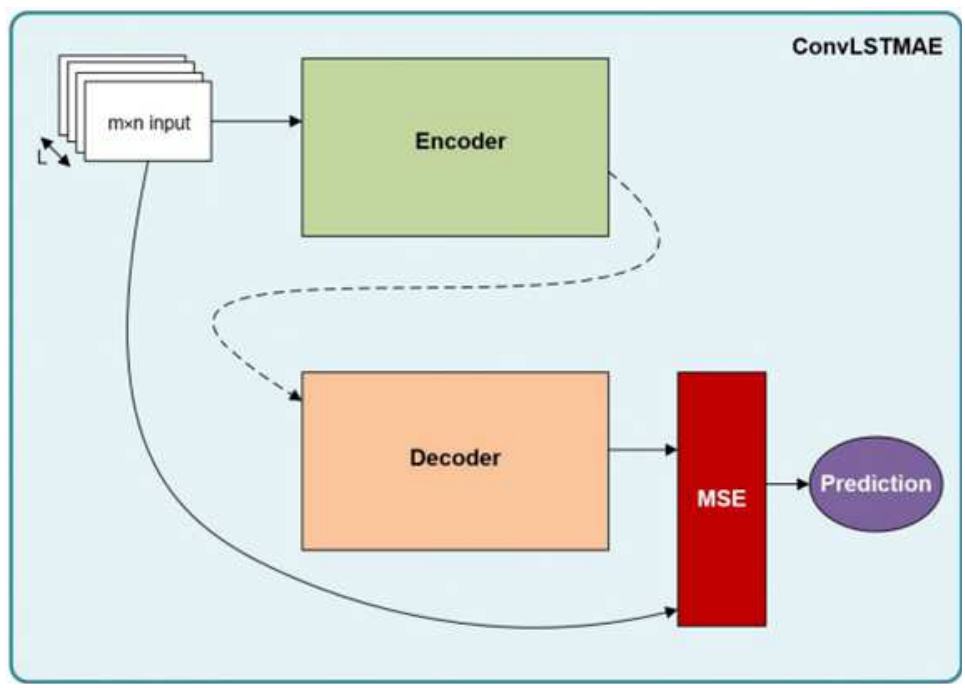

Fig. 6: The ConvLSTMAE architecture

We assumed that data is presented by Tensor $X \in R^{P \times M \times N}$, which there are $P$ measurements in an $M \times N$ grid. As it was mentioned, granular-level patterns do exist in 
daily behavior. Zhang et al. [25] describes these patterns as movement segments, which can be represented by cumulative movement vector.

Till now, to the best of our knowledge, ConvLSTM-based architectures have been used to analyze and perform anomaly detection on videos and images with great success [26] and it is the first time these cells have been leveraged for anomaly detection in behavior.

\section{Experimental Results}

In this section, we present the experimental results of the ConvLSTMAE model and compare it to other state-of-the-art methods. Before getting into the results, we describe our Experimental Setup.

\subsection{Experimental setup}

For the sake of evaluation, we split the datasets into train and test sets, however it is divided with a fixed time period like day preserves the spatiotemporal characteristics in the data [27]. We also partitioned the apartments layouts, illustrated in Fig. 4 and Fig. 5, into a grid, such that each resulting cell hosts a single sensor. In order to implement proposed architecture, we have deployed Keras Deep Learning library's implementation of the CNNs and LSTM [28].

In order to measure our method's performance, the following metrics are utilized: Precision, Recall, Specificity and F-score. We also represent the receiver operating characteristic (ROC) curve, its area under curve (AUC) and precision-recall plot for different threshold values. As denoted by a set of equations in (3), Recall or True Positive (TP) rate is the probability that an actual positive (here the abnormal class) will be predicted as positive, while the true negative (TN) rate (also called specificity) is the probability that an actual negative (here the normal class) will be predicted as negative. On the other hand, Precision measures the number of positive class predictions that actually belong to the positive class. Finally, F-score provides a single score that balances both the concerns of precision and recall in one number.

Anomaly detection performance is evaluated by Recall and Specificity. While Recall reflects the method's capability to detect abnormal instances, Specificity shows correctly identified normal behavior. Thus, Recall and Specificity together, demonstrates method's performance and its potential to correctly discriminate between anomalies and normal patterns. Recall and False Positive (FP) Rate are plotted against each other as ROC curves and provide a principled mechanism to explore operating point tradeoffs. Precision-Recall plots are more informative than ROC curves when evaluating binary classifiers on imbalanced data. 


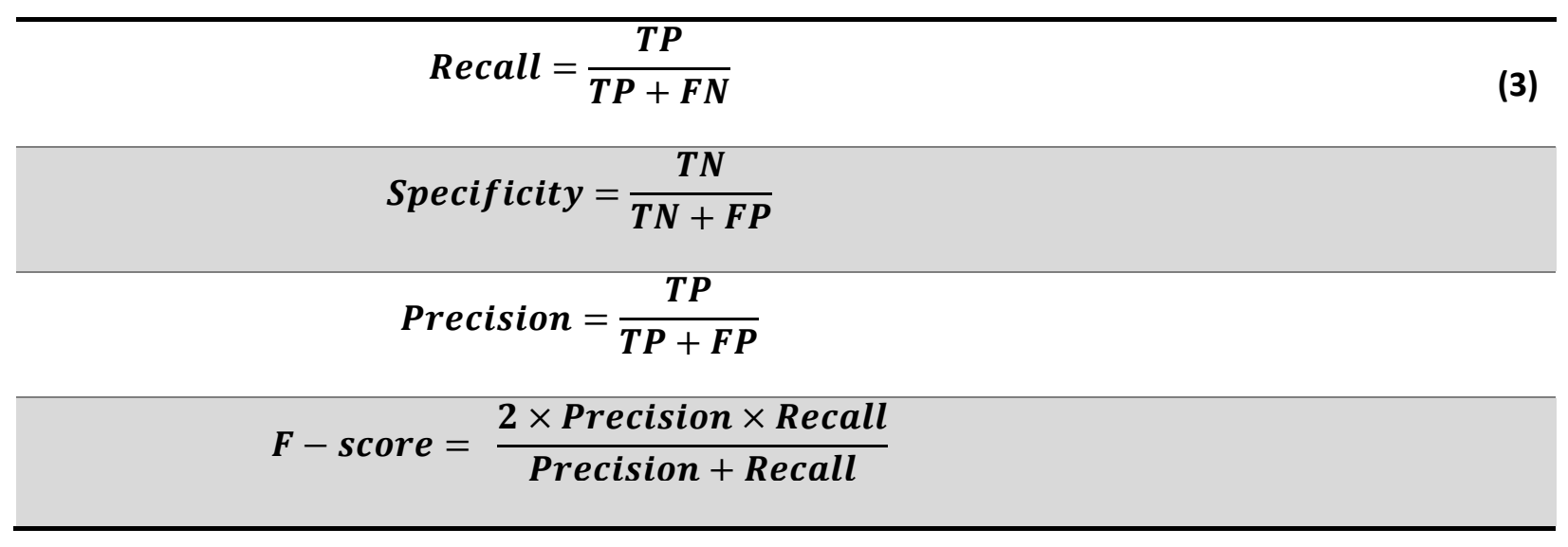

\subsection{Evaluation of ConvLSTMAE}

In this section, we represent the ConvLSTMAE's performance and compare it to the other state-of-the-art methods including OCSVM [29], CNN-LSTM [14] and LSTMAE [24]. In both cases, networks are first trained with normal behavior data and then tested with anomalous data, instances with high reconstruction error (above a certain threshold that was set empirically) were considered abnormal. As we previously mentioned, three possible anomalies have been generated in order to determine ConvLSTMAE's capabilities. Results on the Aruba dataset are depicted in Table I and II, while the results corresponding the Kyoto dataset are provided in Table III.

The results regarding the disturbance in sleep anomaly are demonstrated in Table I. The results show that OCSVM (specificity of 0.91 ) outperforms all the other methods due to the fact that these anomalies are not temporal and completely out of place. However, since OCSVM is not fit to process time-series data, its performance on the repeat anomaly leaves much to be desired. In the disturbance in sleep anomaly, ConvLSTMAE (with specificity of 0.82) outperformed LSTM (specificity of 0.71) and LSTMAE (specificity of 0.73). The results suggest that ConvLSTMAE is capable of extracting meaningful features even in data that is not inherently spatiotemporal, compared to other state-of-the-art methods. These methods also fail in giving good precision results ( 0.035 and 0.03 respectively), and their f-score (0.068 and 0.060) is considerably lower than ConvLSTMAE (f-score of 0.095), indicating ConvLSTMAE is capable of differentiating between normal and abnormal behavior. 
Table I: The performance comparison among discussed models for the 'disturbance in sleep' anomaly

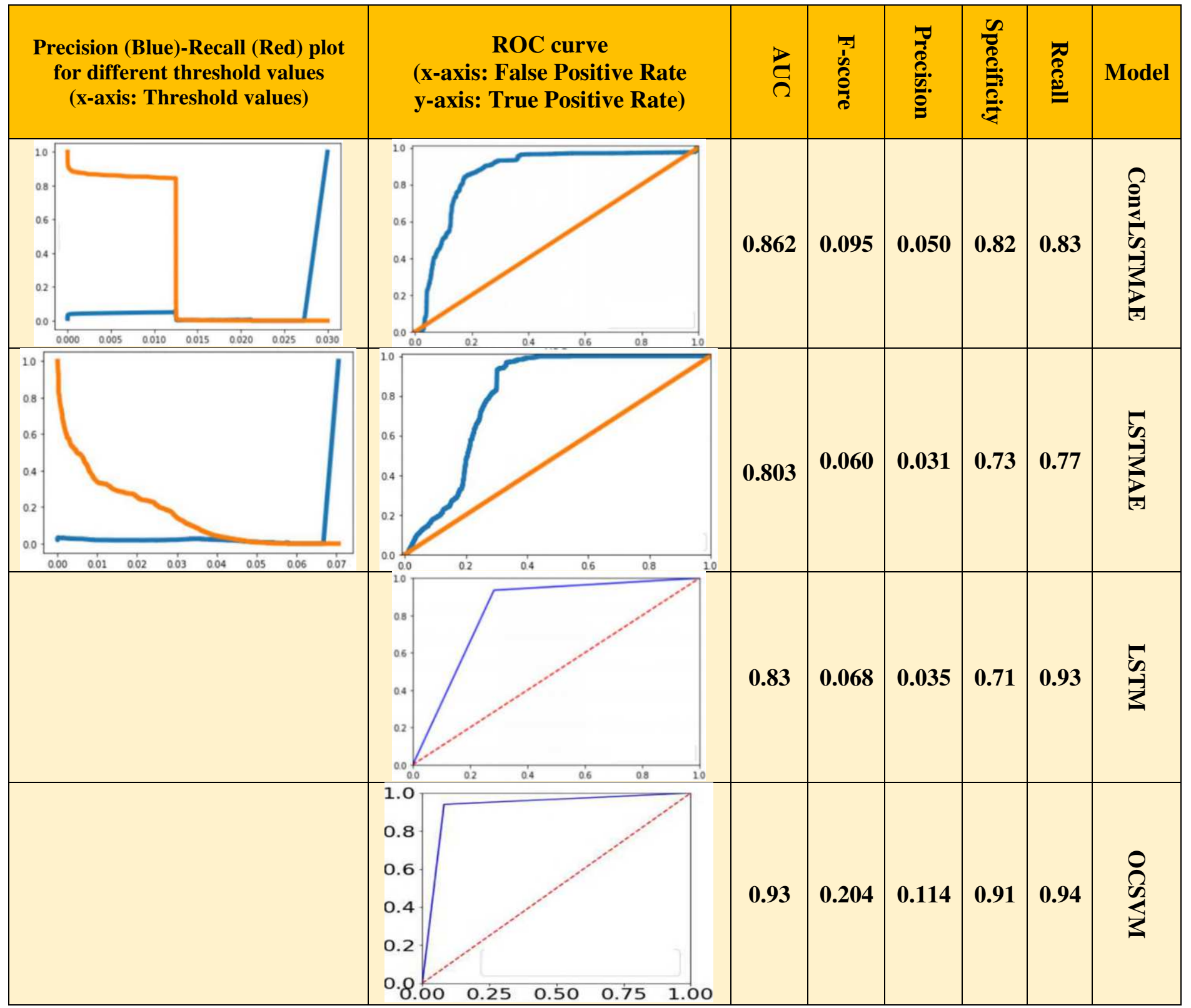


Moreover, the results for the repetition anomaly is represented in Table II. In this type of anomaly, since it is highly correlated to previous instances, OCSVM performs poorly, which is expected. Our method achieved the highest f-score (f-score of 0.12 , compared to 0.073, 0.041 and 0.066 for LSTMAE, LSTM and OCSVM, respectively), falling behind on recall (LSTMAE achieved highest recall, followed by ConvLSTMAE, LSTM and OCSVM, respectively), but again higher precision and specificity rates are indicative of significant improvement over other methods. OCSVM low precision and recall (0.037 and 0.36, respectively) is indicative of failing to learn class specific features to correctly differentiate between anomaly and normal behavior. $\mathrm{CNN}$ is able to detect changes in feature patterns implicitly, due to the fact that each activity is formed by steps and these steps are captured by motion sensors throughout the home. As an example, in having dinner in midnight, one must first go to the kitchen, then start the oven and prepare food. LSTM also captures repetition related activities. Our proposed method is not able to detect anomalies in specialized activities like adding too much seasoning to food, or having a bland meal since patient forgot to add salt, confusing the location of a certain item or leaving kitchen utilities on, due to the fact that there are no sensors pertaining to these activities in the Aruba dataset and future research are expected to address this problem. Furthermore, our method may be detecting abnormal behavior with delay, if there are gradual deterioration in patient's health, since the reconstruction error needs to reach a minimum amount. Having said that, this problem can be somewhat resolved with fine-tuning the network for each individual.

Finally, the results corresponding the confusion anomaly is provided in Table III. In this anomaly, ConvLSTMAE offers the best result (AUC of 0.903); however, its performance is within the margin of error compared to LSTMAE. Convolutional layer's contribution was more evident in the Aruba data as compared to the Kyoto data, since our method requires normal daily activities in order to detect anomalies based on time of occurrence. Hence it cannot fully demonstrate its power on the Kyoto dataset. That being said, our method's advantages are that it does not need labels to distinguish anomalies (as it was mentioned before, in real-world datasets, labels are not usually available) and floating threshold which can be utilized as a fine-tuning method. In OCSVM, anomaly ratio must be specified beforehand which again is not a valid assumption. 
Table II: The performance comparison among discussed models for the 'repetition' anomaly

\begin{tabular}{|c|c|c|c|c|c|c|c|}
\hline $\begin{array}{l}\text { Precision (Blue)-Recall (Red) Plot } \\
\text { for different threshold values } \\
\text { (x-axis: Threshold values) }\end{array}$ & $\begin{array}{c}\text { ROC Curve } \\
\text { (x-axis: False Positive Rate } \\
\text { y-axis: True Positive Rate) }\end{array}$ & $\stackrel{\vec{R}}{\Omega}$ & है & ". & 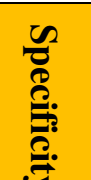 & 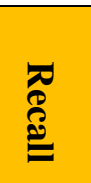 & Model \\
\hline 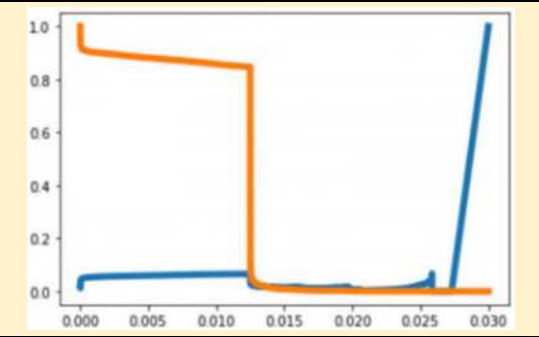 & (2.) & 0.853 & 0.12 & 0.065 & 0.84 & 0.84 & 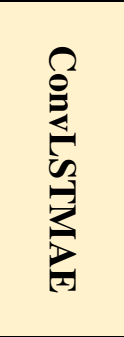 \\
\hline 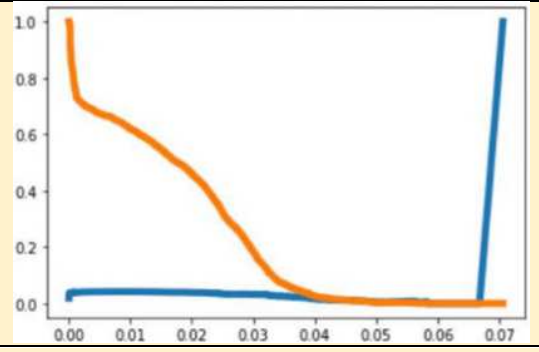 & . & 0.836 & 0.073 & 0.038 & 0.67 & 0.98 & 铍 \\
\hline & $\begin{array}{lllll}0.6 & 0.6 & 0\end{array}$ & 0.65 & 0.041 & 0.021 & 0.57 & 0.71 & 告 \\
\hline & 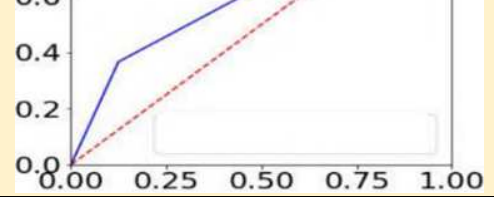 & 0.62 & 0.066 & 0.036 & 0.87 & 0.36 & 3 \\
\hline
\end{tabular}


Table III: The performance comparison among discussed models for the 'confusion' anomaly

\begin{tabular}{|c|c|c|c|c|c|c|c|}
\hline $\begin{array}{c}\text { Precision (Blue)-Recall (Red) } \\
\text { Plot for different threshold } \\
\text { values } \\
\text { (x-axis: Threshold values) }\end{array}$ & $\begin{array}{c}\text { ROC Curve } \\
\text { (x-axis: False Positive Rate } \\
\text { y-axis: True Positive Rate) }\end{array}$ & $\vec{\Omega}$ & $\begin{array}{l}\text { Tे } \\
\dot{\omega} \\
\hat{0} \\
0\end{array}$ & 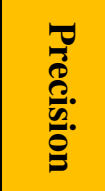 & 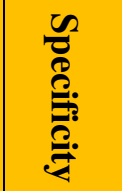 & 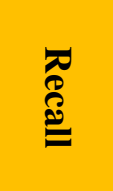 & Model \\
\hline (10) & (1) & 0.903 & 0.688 & $\mathbf{0 . 5 3 8}$ & 0.793 & 0.957 & 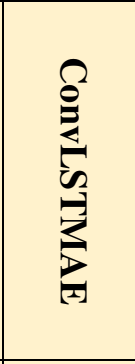 \\
\hline (10 & $\int_{0.2}^{0.6 .5}$ & $\mathbf{0 . 8 9 9}$ & 0.687 & $\mathbf{0 . 5 3 6}$ & 0.792 & 0.955 & 要 \\
\hline & & 0.81 & 0.61 & 0.48 & 0.78 & 0.81 & $\frac{5}{3}$ \\
\hline & 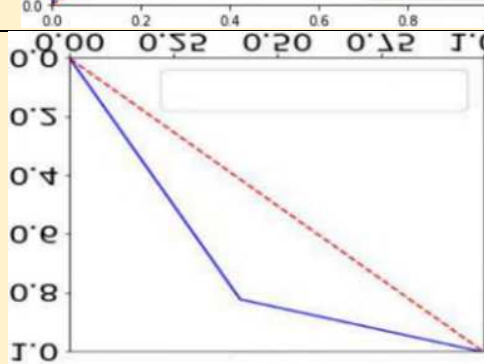 & $\mathbf{0 . 7 0}$ & 0.47 & $\mathbf{0 . 3 3}$ & 0.58 & 0.81 & $\begin{array}{l}0 \\
0 \\
3 \\
3 \\
3\end{array}$ \\
\hline
\end{tabular}




\section{Conclusions}

This paper introduces a novel method for detecting abnormal behavior pertaining to dementia in elderly people in smart homes. We have utilized ConvLSTMAE in order to process spatiotemporal data. Results on anomaly detection on the dataset shows tremendous improvement over the state-of-the-art methods. Tackling this problem with AE means our method can be generalized to detect other abnormal behaviors of elderly people whom suffer from dementia.

\section{Declaration of competing Interest}

The authors declare that they have no conflicts of interest.

\section{Authorship contribution statement}

Ehsan Nazerfard: Supervision, Writing - review \& editing. Zahra Atashgahi: Writing, Investigation. Alireza Nadali: Writing - review \& editing

\section{References}

[1] M. Vijayan and P. Reddy, "Stroke, Cascular Dementia, and Alzheimer's Disease: Molecular Links," Journal of Alzheimer's Disease, vol. 54, no. 2, pp. 427-443, 2016.

[2] E. Soleimani and E. Nazerfard, "Cross-Subject Transfer Learning in Human Activity Recognition Systems using Generative Adversarial Networks," Neurocomputing, vol. 426, pp. 26-34, 2021.

[3] H. Shahabadi, A. Fatehi, A. Nadali and S. M.A, "A Novel Method For Designing Transferable Soft Sensors And Its Application," ArXive preprint, available at: https://arxiv.org/abs/2008.02186, 2020.

[4] M. Ahmed, A. Mahmood and M. Islam, "A survey of anomaly detection techniques in financial domain," Future Generation Computer Systems, 2015.

[5] Q. Deng and G. Mei, "Combining self-organizing map and k-means clustering," in IEEE International Conference on Granular Computing, 2009.

[6] J. Shin, B. Lee and K. Park, "Detection of abnormal living patterns for elderly living alone," in IEEE Trans. Inf. Technol. Biomed, 2011.

[7] D. Cook and M. Schmitter-Edgecombe, "Assessing the Quality of Activities in a Smart Environment," Methods of Information in Medicine, vol. 48, pp. 480-485, 2009.

[8] N. Hammerla, J. Fisher, P. Andras, L. Rochester, R. Walker and T. Plotz, "PD disease state assessment in naturalistic environments using deep learning," in Proceedings of the Twenty-Ninth AAAI Conference on Artificial Intelligence, 2015. 
[9] D. Lara and M. Labrador, "A mobile platform for real-time human activity recognition," in IEEE Consumer Communications and Networking Conference (CCNC), 2012.

[10] J. Yang, M. Nguyen, P. San, X. Li Li and S. Krishnaswamy, "Deep convolutional neural networks on multichannel time series for human activity recognition," in Proceedings of the 24th International Conference on Artificial Intelligence, 2015.

[11] M. Zeng, L. Nguyen, B. Yu and O. J. Mengshoel, "Convolutional Neural Networks for Human Activity Recognition using Mobile Sensors," in Sixth International Conference on Mobile Computing, Applications and Services, 2014.

[12] C. Ronao and S. Cho, "Human activity recognition with smartphone sensors using deep learning neural networks," Expert Systems with Applications, vol. 59, pp. 235-244, 2016.

[13] F. Ordóñez and D. Roggen, "Deep Convolutional and LSTM Recurrent Neural Networks for Multimodal Wearable Activity Recognition," Advances on Data Transmission and Analysis for Wearable Sensors Systems, vol. 16, p. 1, 2016.

[14] D. Arifoglu and A. Bouchachia, "Detection of abnormal behaviour for dementia sufferers using Convolutional," Artificial Intelligence In Medicine, vol. 94, pp. 88-95, 2019.

[15] D. Cook, A. Crandall, B. Thomas and N. Krishnan, "CASAS: A Smart Home in a Box," Computer, vol. 46, p. 6269, 2013.

[16] S. Hochreiter and J. Schmidhuber, "Long Short-Term Memory," Neural Computation, vol. 9(8), pp. 1735-17880, 1997.

[17] J. Elman, "Finding structure in time," Cognitive science, vol. 14, no. 2, pp. 179-211, 1990.

[18] Y. LeCun and Y. Bengio, "Convolutional networks for images, speech, and time series," in The handbook of brain theory and neural networks, Cambridge, MIT Press, 1998, pp. 255-258.

[19] Shi et al., "Convolutional LSTM Network: a machine learning approach for precipitation nowcasting," in Proceedings of the 28th International Conference on Neural Information Processing Systems, 2015.

[20] I. Goodfellow, Y. Bengio and A. Courville, Deep Learning, The MIT Press, 2016.

[21] M. Gochoo, T. Tan, S. Huang, S. Liu and F. Alnajjar, "DCNN-based elderly activity recognition using binary sensors," in M. Gochoo, T. Tan, S. Huang, S. Liu and F. S. Alnajjar, "DCNN-based elderly actilnternational Conference on Electrical and Computing Technologies and Applications (ICECTA), 2017.

[22] D. Arifoglu and A. Bouchachia, "Activity Recognition and Abnormal Behaviour Detection with Recurrent Neural Networks," Procedia Computer Science, vol. 110, pp. 86-93, 2017. 
[23] J. Saives, C. Pianon and G. Faraut, "Activity Discovery and Detection of Behavioral Deviations of an Inhabitant From Binary Sensors," IEEE Transactions on Automation Science and Engineering, vol. 12, pp. 1211-1224, 2015.

[24] P. Malhorta, A. Ramakrishnan, G. Anand, L. Vig, P. Agarwal and G. Shroff, "LSTM-based EncoderDecoder for Multi-sensor Anomaly Detection," ArXive preprint, available at :https://arxiv.org/abs/1607.00148, 2016.

[25] T. Zhang, W. Fu, J. Ye and M. Fischer, "Learning movement patterns of the occupant in smart home," Journal of Ambient Intelligence and Humanized Computing, vol. 8, pp. 133-146, 2017.

[26] J. Mede and A. Savakis, "Anomaly Detection in Video Using Predictive Convolutional Long ShortTerm," ArXive preprint, available at: https://arxiv.org/abs/1612.00390, 2016.

[27] N. Suryadevara, S. Mukhopadhyay, R. Wang and R. Rayudu, "Forecasting the behavior of an elderly using wireless sensors data in a smart home," Engineering Applications of Artificial Intelligence, vol. 26, no. 10, pp. 2641-2652, 2013.

[28] C. François, "Keras," 2015. [Online]. Available: https://github.com/keras-team/keras.

[29] V. Jakkula and D. Cook, "Detecting Anomalous Sensor Events in Smart Home Data for Enhancing the Living Experience," in Artificial Intelligence and Smarter Living: The Conquest of Complexity, 2011.

[30] T. Van Kasteren, G. Englebienne and B. J. A. Kröse, "Human Activity Recognition from Wireless Sensor Network Data: Benchmark and Software," in Activity Recognition in Pervasive Intelligent Environments. Atlantis Ambient and Pervasive Intelligence, vol 4., Atlantis Press, 2011, pp. 165186. 